\title{
Pneumococcal Vertebral Osteomyelitis and Psoas Abscess in a Patient with Systemic Lupus Erythematosus Disclosing Positivity of Pneumococcal Urinary Antigen Assay
}

\author{
Masami Matsumura ${ }^{1}$, Kiyoaki Ito ${ }^{2}$, Rika Kawamura ${ }^{2}$, Hiroshi Fujii ${ }^{2}$, Ryo Inoue ${ }^{2}$, \\ Kazunori Yamada ${ }^{2}$, Masakazu Yamagishi ${ }^{3}$ and Mitsuhiro Kawano ${ }^{2}$
}

\begin{abstract}
A 53-year-old woman with systemic lupus erythematosus presented with a 3-day history of fever and coughing. Diagnosis of pneumococcal bronchitis was made based on symptoms and positivity of pneumococcal urinary antigen test. On day 3, severe low back pain acutely occurred. Pneumococcal vertebral osteomyelitis and psoas abscess was diagnosed 17 days later by yield of penicillin-susceptible $S$. pneumoniae strain in blood cultures and drainage fluid. Although pneumococcal urinary antigen test is a useful tool for the diagnosis of pneumococcal pneumonia, we should consider the possibility of pneumococcal infections other than pneumonia or overwhelming bacteremia in immunosuppressive patients when urinary antigen test is positive.
\end{abstract}

Key words: pneumococcal vertebral osteomyelitis, psoas abscess, systemic lupus erythematosus, Streptococcus pneumoniae, pneumococcal urinary antigen test

(Intern Med 50: 2357-2360, 2011)

(DOI: 10.2169/internalmedicine.50.5863)

\section{Introduction}

Streptococcus pneumoniae infections have been a significant cause of morbidity and mortality, especially children and the elderly. S. pneumoniae commonly causes pneumonia, otitis media, sinusitis, and meningitis (1). On the other hand, pneumococcal vertebral osteomyelitis is very uncommon. The pneumococcal urinary antigen test is useful tool for the diagnosis of pneumococcal pneumonia $(2,3)$. Moreover, the usefulness of this test for the diagnosis of pneumococcal infections other than pneumonia such as acute otitis media, pericarditis, and bacteremia has been reported (4-6).

We describe a case of pneumococcal vertebral osteomyelitis and psoas abscess in a patient with systemic lupus erythematosus (SLE) disclosing a positive result of pneumococcal urinary antigen test in the early clinical course and discuss interpretation of positivity of the pneumococcal urinary antigen test.

\section{Case Report}

A 53-year-old woman who had a 3-day history of fever was admitted to another hospital. She had been in good health until 3 days before admission, when a fever of 39.0 ${ }^{\circ} \mathrm{C}$ and coughing developed. Lupus nephritis had been diagnosed 30 years previously, and remission of nephritis had been induced with an initial dose of $30 \mathrm{mg}$ per day of oral prednisolone. She had had corticosteroid-induced diabetes mellitus for 14 years. She had undergone surgery for lumbar disc herniation at the ages of 47 and 48, and coronary artery bypass grafting at the age of 49 . She had not been vaccinated against $S$. pneumoniae. She was receiving $8 \mathrm{mg}$ per day of prednisolone, $750 \mathrm{mg}$ per day of metformin, $100 \mathrm{mg}$ per day of aspirin, $40 \mathrm{mg}$ per day of furosemide, and $10 \mathrm{mg}$ per day of atorvastatin. Influenza, bronchitis, or pneumonia was suspected because of fever and coughing. Blood tests showed leukocyte count $15,700 / \mu \mathrm{L}$ and C-reactive protein

\footnotetext{
${ }^{1}$ Research Center for Medical Education, Kanazawa University Graduate School of Medicine, Japan, ${ }^{2}$ Division of Rheumatology, Department of Internal Medicine, Kanazawa University Graduate School of Medicine, Japan and ${ }^{3}$ Division of Cardiology, Department of Internal Medicine, Kanazawa University Graduate School of Medicine, Japan

Received for publication May 21, 2011; Accepted for publication July 13, 2011

Correspondence to Dr. Masami Matsumura, mmatsu@spacelan.ne.jp
} 
Table 1. Laboratory Findings

$\begin{array}{ll}\text { WBC }(/ \mu \mathrm{L}) & 23,100 \\ \text { Neutrophils }(\%) & 97 \\ \text { Lymphocytes }(\%) & 1.0 \\ \text { Monocytes }(\%) & 1.0 \\ \text { Myelocytes }(\%) & 1.0 \\ \text { Hemoglobin }(\mathrm{g} / \mathrm{dL}) & 12.1 \\ \text { Platelet }(/ \mu \mathrm{L}) & 364,000 \\ \text { Howell-Jolly bodies } & (-) \\ \text { AST (IU/L) } & 10 \\ \text { ALT (IU/L) } & 15 \\ \text { creatinine }(\mathrm{mg} / \mathrm{dL}) & 0.87 \\ \text { Na (mEq/L) } & 140 \\ \text { K (mEq/L) } & 4.7 \\ \text { Cl (mEq/L) } & 102 \\ \text { Hemoglobin A1C }(\%) & 7.4\end{array}$

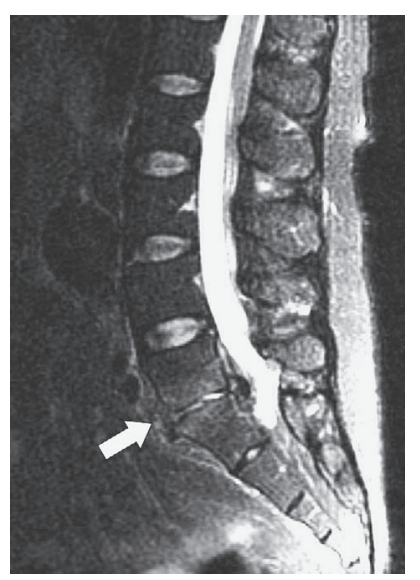

Figure 1. Sagittal magnetic resonance image of the lumbar spine showed L5/S1 osteomyelitis (arrow).

$5.1 \mathrm{mg} / \mathrm{dL}$. Rapid diagnostic test for influenza A and B was negative. Although urine immunochromatographic membrane assay for S. pneumoniae (Binax $\mathrm{NOW}^{\circledR} \quad$ Streptococcus pneumoniae, Binax Inc., Scarborough, ME, USA) was positive, chest X-ray showed no abnormalities. Acute bronchitis due to $S$. pneumoniae was diagnosed. Blood culture was not performed. One gram per day of intravenous pazufloxacin mesilate was started. On day 3, severe low back pain acutely occurred and she could not walk because of the pain. She was eager to increase the prednisolone dosage to alleviate the excruciating back pain. On day 6 , the dose of prednisolone was increased to $40 \mathrm{mg}$ per day and was somewhat effective. She could walk and the fever subsided. On day 10, administration of pazufloxacin mesilate was discontinued. However the low back pain persisted. She was transferred to our hospital for further examination and treatment on day 20. On physical examination, temperature was $38.0^{\circ} \mathrm{C}$, blood pressure $166 / 96 \mathrm{mmHg}$, pulse 75 beats per minute, and respiratory rate 20 per minute. She complained of back pain. Nuchal rigidity was absent. Chest and abdominal examinations showed no abnormalities. Psoas signs were negative bilaterally. Tenderness over the spine was not observed. Laboratory findings are shown in Table 1. No

$\begin{array}{ll}\text { CRP }(\mathrm{mg} / \mathrm{dL}) & 5.5 \\ \mathrm{IgG}(\mathrm{mg} / \mathrm{dL}) & 1,100 \\ \mathrm{IgA}(\mathrm{mg} / \mathrm{dL}) & 136 \\ \mathrm{IgM}(\mathrm{mg} / \mathrm{dL}) & 22 \\ \mathrm{CH} 50(\mathrm{U} / \mathrm{mL})(\text { normal 32-47) } & 59 \\ \mathrm{C} 3(\mathrm{mg} / \mathrm{dL})(\text { normal } 65-135) & 145 \\ \text { C4 (mg/dL) (normal 13-35) } & 30 \\ \text { Antinuclear antibodies } & \times 20 \\ & \text { speckled } \\ \text { Autoantibodies to anti-Sm } & (-) \\ \text { Autoantibodies to dsDNA } & (-) \\ & \\ \text { Urinalysis } & \\ \quad \text { Proteinuria } & (-) \\ \text { Occult blood } & (-) \\ \text { Glycosuria } & (-)\end{array}$

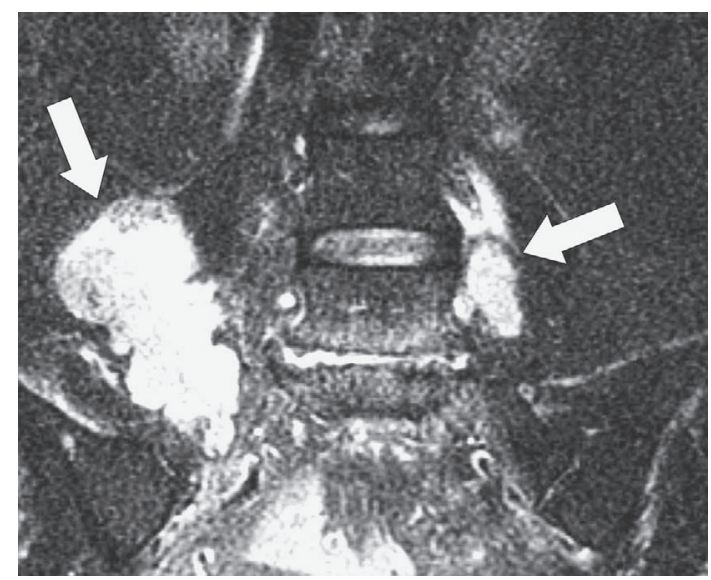

Figure 2. Coronal magnetic resonance image of the lumbar spine showed bilateral psoas abscess (arrows).

Howell-Jolly bodies were observed in peripheral blood smear. Systemic Lupus Erythematosus Disease Activity Index (SLEDAI) (7) was zero. Magnetic resonance imaging (MRI) of the lumbar spine showed L5/S1 osteomyelitis (Fig. 1) and bilateral psoas abscess (Fig. 2). On day 2, CTguided drainage of the psoas abscess was performed bilaterally and purulent fluid was obtained. Gram staining of the purulent material showed gram-positive cocci in chains. After the procedure, two grams per day of intravenous vancomycin was started. Prednisolone was tapered to $7.5 \mathrm{mg}$ per day. Two sets of blood culture and drainage purulent fluid yielded penicillin-susceptible $S$. pneumoniae strain. On day 6 , vancomycin was changed to 2 grams per day of intravenous ceftriaxone. On day 13, drainage tubes were taken out. Repeat MRI on day 22 showed complete resolution of the psoas abscess, although the finding of L5/S1 osteomyelitis did not change. On day 45, laboratory values were as follows: leukocyte count $7,900 / \mu \mathrm{L}$, C-reactive protein $0.1 \mathrm{mg} /$ dL, and hemoglobin A1C 6.5\% (Fig. 3). On day 63, ceftriaxone was changed to $200 \mathrm{mg}$ per day of oral cefpodoxime proxetil and she was discharged with good health status. She was vaccinated with 23 -valent pneumococcal vaccine. Severe infections were not observed during the four year 


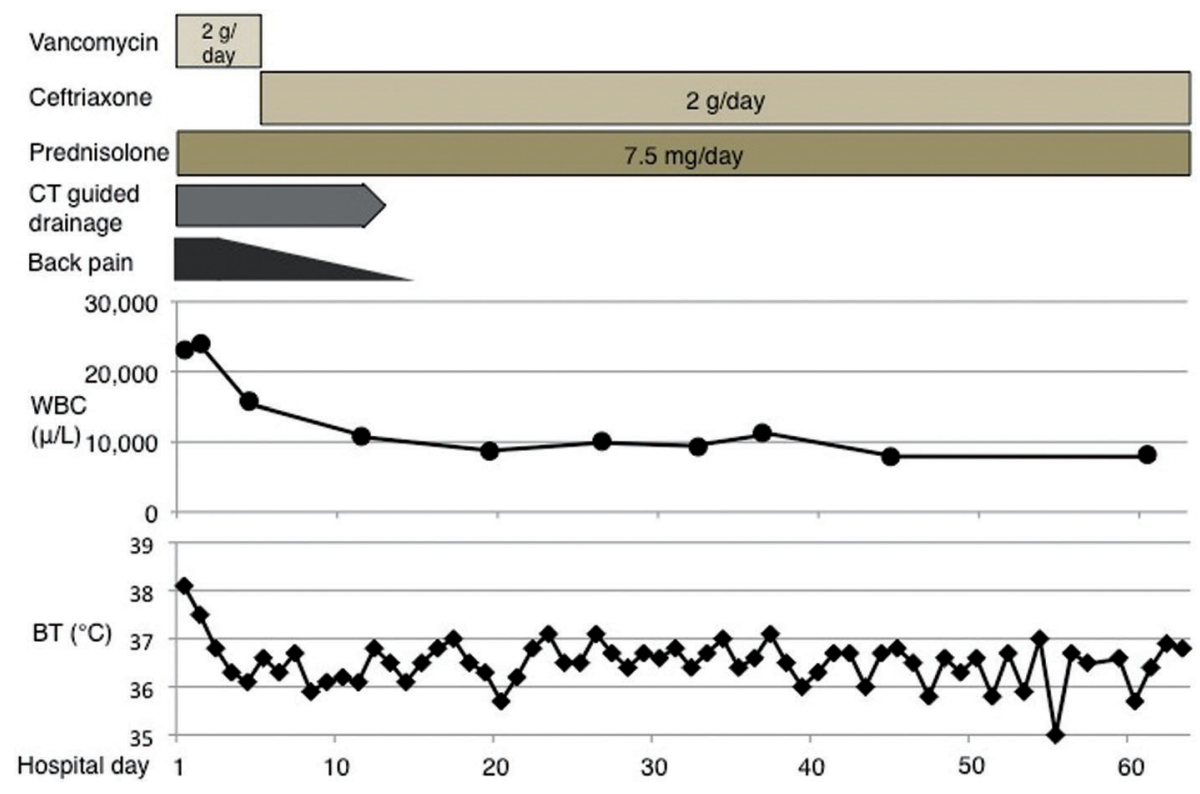

Figure 3. Clinical course of the patient.

follow-up.

\section{Discussion}

S. pneumoniae is a gram-positive coccus which is protected from complement-mediated opsonization and phagocytosis by the presence of a polysaccharide capsule. $S$. pneumoniae generally infects the middle ear, sinuses, trachea, bronchi, and lungs by direct spread of organisms from colonization in nasopharynx and causes infections of the central nervous system, and rarely heart valves, peritoneal cavity, joint, and bone hematogenously. However, S. pneumoniae can penetrate the mucosal barrier directly (1) and overwhelming pneumococcal bacteremia can occur in splenectomized patients. The spleen can clear unopsonized $S$. pneumoniae from the circulation by the slow blood flow though the spleen resulting in extended contact with reticuloendothelial cells in the cords of Billroth (1). Functional asplenia in patients with SLE associated with overwhelming pneumococcal bacteremia was described (8). Moreover, overwhelming pneumococcal bacteremia was reported in a previous healthy patient (9).

Vertebral osteomyelitis is a life-threatening infection and often recurs. Reported causative pathogens of vertebral osteomyelitis were Staphylococcus aureus, coagulase-negative staphylococci, streptococci, Escherichia coli, Pseudomonas aeruginosa, Proteus mirabilis, Klebsiella pneumoniae, and salmonella species (10). Reported significant risk factors in 72 vertebral osteomyelitis patients were previous focal infection and/or bacteremia (58.3\%), diabetes mellitus (23.6\%), history of spinal surgery $(23.6 \%)$, and intravenous drug use (12.5\%) (11). S. pneumoniae is a rare causative pathogen in vertebral osteomyelitis. Turner et al reviewed 28 cases with pneumococcal vertebral osteomyelitis, none of them SLE, including their 8 cases (12). Eight of the twenty-eight cases
(28.6\%) had a history of recent respiratory tract infection. In the present case, fever and coughing in the early clinical course were noted. The respiratory tract might have been the portal of entry for S. pneumoniae and it spread to vertebral bone hematogenously. On the other hand, rapid hematogenous spread of $S$. pneumoniae following direct penetration of the mucosal barrier was undeniable in this case.

The pneumococcal urinary antigen assay is an in vitro immunochromatographic assay for the detection of cell wall polysaccharide antigen on $S$. pneumoniae in the urine of patients with pneumococcal pneumonia (2). Reported sensitivity and specificity for pneumococcal pneumonia were high at $64.3-82 \%$ and $77-98.8 \%$, respectively $(2,3)$. Moreover, diagnoses of pneumococcal infections using pneumococcal urinary antigen test with specimens other than urine such as middle ear fluids, nasopharyngeal secretions, pleural fluids, pericardial fluid, and blood cultures have been reported $(4-6,13)$. Although pneumococcal urinary antigen tests are significantly greater in patients with pneumonia (87\%) than in patients with other pneumococcal infections (70\%) (14), positivity of pneumococcal urinary antigen test can indicate pneumococcal infections other than pneumonia. Reported sensitivity for pneumococcal bacteremia was high at $82 \%$ (14). Moreover, we should clarify the past history of pneumococcal pneumonia when pneumococcal urinary antigen test is positive. Andreo et al reported that the positivity of pneumococcal urinary antigen test persisted for one month in $18(52.9 \%)$ of the 34 patients and for 4 months in 6 patients after the diagnosis of pneumococcal pneumonia (15). In the present case, a positive result of pneumococcal urinary antigen test early in the clinical course might lead to early closure of the physician's thinking process for the etiology of back pain and subsequently delay the diagnosis of vertebral osteomyelitis.

Infectious complications are still a major cause of mor- 
bidity and mortality in patients with SLE (16-18). Causative pathogens of infections in lupus patients are gram-positive cocci, gram-negative bacilli, and other organisms of opportunistic infections $(17,18)$. Past or current use of corticosteroids (17) and disease activity (18) are significantly associated with infections. In addition, intrinsic immunological defects including low immunoglobulin levels, hypocomplementemia, impaired cell-mediated immunity, and impeded opsonized bacteria clearance in the reticuloendothelial system are considered as other predisposing factors. Wu et al retrospectively investigated 11 cases of osteomyelitis in lupus patients, and showed that major causative pathogens were Salmonella and S. aureus. Significant predisposing factors were SLEDAI sore $>4$, coexistent underlying systemic disease, chronic renal disease, and aggressive immunosuppressive therapy (19). In the present case, pneumococcal bacteremia was observed, however, functional asplenia was not evident. The predisposition to pneumococcal infection was thought to have been multifactorial, including the immune defects of SLE itself, prolonged corticosteroid use, diabetes mellitus, and history of spinal surgery.

In summary, this is a case report of pneumococcal vertebral osteomyelitis and psoas abscess in a patient with SLE disclosing positivity of pneumococcal urinary antigen test. Urinary antigen test for $S$. pneumoniae is a useful tool for the diagnosis of pneumococcal infections, however, we should consider the possibility of pneumococcal infections other than pneumonia or overwhelming bacteremia in immunosuppressive patients when the urinary antigen test is positive.

The authors state that they have no Conflict of Interest (COI).

\section{References}

1. Musher DM. Streptococcus pneumoniae. In: Principles and Practice of Infectious Disease. 6th ed. Mandell GL, Bennett JE, Dolin R, Eds. Elsevier, Philadelphia, 2005: 2392-2411.

2. Burel E, Dufour P, Gauduchon V, Jarraud S, Etienne J. Evaluation of a rapid immunochromatographic assay for detection of Streptococcus pneumoniae antigen in urine samples. Eur J Clin Microbiol Infect Dis 20: 840-841, 2001.

3. Genné D, Siegrist HH, Lienhard R. Enhancing the etiologic diagnosis of community-acquired pneumonia in adults using the urinary antigen assay (Binax NOW). Int J Infect Dis 10: 124-128, 2006.

4. Gisselsson-Solén M, Bylander A, Wilhelmsson C, Hermansson A, Melhus A. The Binax NOW test as a tool for diagnosis of severe acute otitis media and associated complications. J Clin Microbiol 45: 3003-3007, 2007.

5. Nakagawa C, Kasahara K, Yonekawa S, et al. Purulent pericarditis due to Streptococcus pneumoniae diagnosed by pneumococcal urinary antigen assay and $16 \mathrm{~S}$ rDNA sequence of the pericardial fluid. Intern Med 49: 1653-1656, 2010.

6. Petti CA, Woods CW, Reller LB. Streptococcus pneumoniae antigen test using positive blood culture bottles as an alternative method to diagnose pneumococcal bacteremia. J Clin Microbiol 43: 2510-2512, 2005.

7. Bombardier C, Gladman DD, Urowitz MB, et al. Derivation of the sledai. A disease activity index for lupus patients. Arthritis Rheum 35: 630-640, 1992.

8. Hühn R, Schmeling H, Kunze C, Horneff G. Pneumococcal sepsis after autosplenectomy in a girl with systemic lupus erythematosus. Rheumatology 44: 1586-1588, 2005.

9. Nakamura H, Saitou M, Kinjo S, et al. Overwhelming pneumococcal bacteremia revealed by a peripheral blood smear in a 74 year-old healthy woman. Intern Med 46: 303-306, 2007.

10. McHenry MC, Easley KA, Locker GA. Vertebral osteomyelitis: long-term outcome for 253 patients from 7 Cleveland-area hospitals. Clin Infect Dis 34: 1342-1350, 2002.

11. Colmenero JD, Jiménez-Mejías ME, Sánchez-Lora FJ, et al. Pyogenic, tuberculous, and brucellar vertebral osteomyelitis: a descriptive and comparative study of 219 cases. Ann Rheum Dis 56: 709715, 1997.

12. Turner DPJ, Weston VC, Ispahani P. Streptococcus pneumoniae spinal infection in Nottingham, United Kingdom: not a rare event. Clin Infect Dis 28: 873-881, 1999.

13. Porcel JM, Ruiz-González A, Falguera M, et al. Contribution of a pleural antigen assay (Binax NOW) to the diagnosis of pneumococcal pneumonia. Chest 131: 1442-1447, 2007.

14. Smith MD, Derrington P, Evans R, et al. Rapid diagnosis of bacteremic pneumococcal infections in adults by using the Binax NOW Streptococcus pneumoniae urinary antigen test: a prospective, controlled clinical evaluation. J Clin Microbiols 41: 28102813, 2003.

15. Andreo F, Prat C, Ruiz-Manzano J, et al. Persistence of Streptococcus pneumoniae urinary antigen excretion after pneumococcal pneumonia. Eur J Clin Microbiol Infect Dis 28: 197-201, 2009.

16. Cervera R, Khamashta MA, Font J, et al. Morbidity and mortality in systemic lupus erythematosus during a 10-year period: a comparison of early and late manifestations in a cohort of 1,000 patients. Medicine 82: 299-308, 2003.

17. Gladman DD, Hussain F, Ibañez D, Urowitz MB. The nature and outcome of infection in systemic lupus erythematosus. Lupus 11: 234-239, 2002.

18. Zonana-Nacach A, Camargo-Coronel A, Yañez P, Sánchez L, Jimenez-Balderas FJ, Fraga A. Infections in outpatients with systemic lupus erythematosus: a prospective study. Lupus 10: 505510, 2001.

19. Wu KC, Yao TC, Yeh KW, Huang JL. Osteomyelitis in patients with systemic lupus erythematosus. J Rheumatol 31: 1340-1343, 2004.

(C) 2011 The Japanese Society of Internal Medicine

http://www.naika.or.jp/imindex.html 